\title{
Evaluación de la función sexual femenina antes y después de un procedimiento para la corrección de la incontinencia urinaria de esfuerzo
}

\author{
R. Thiel*, M. Thiel*, M. Dambros*, C. Riccetto*, V. López**, M.E. Rincón***, P. Palma*. \\ *Disciplina de Urología, Universidade Estadual de Campinas, UNICAMP, SP, Brazil. \\ **Servicio de Urología del Hospital Universitario de Caracas, Caracas, Venezuela. \\ ***Instituto Docente de Urologia, Universidad de Carabobo, Valencia, Venezuela.
}

Actas Urol Esp 2006; 30 (3): 315-320

\begin{abstract}
RESUMEN
EVALUACIÓN DE LA FUNCIÓN SEXUAL FEMENINA ANTES Y DESPUÉS DE UN PROCEDIMIENTO PARA LA CORRECCIÓN DE LA INCONTINENCIA URINARIA DE ESFUERZO

La incontinencia urinaria afecta a cerca del $20 \%$ de las mujeres en edad reproductiva y afecta negativamente la calidad de vida y la sexualidad. Entre agosto de 2002 y enero 2004, 30 pacientes portadoras de incontinencia urinaria (IU), respondieron los cuestionarios ICIQ-SF (Internacional Consultation on Incontinente QuestionaireShort Form) y FSFI (Female Sexual Function Index) antes y después del tratamiento. La edad media fue de 43 años (31 a 51 años), con un tiempo de inicio de los síntomas urinarios que varió entre 12 a 53 meses. Participaron pacientes con incontinencia urinaria de esfuerzo (59\%), vejiga hiperactiva idiopática (15\%) e incontinencia urinaria mixta (26\%). Se utilizó el test de Wilcoxon para datos pareados para la evaluación estadística. Los valores de $\mathrm{p} \leq 0,05$ indicaron diferencias significativas de la respuesta sexual (RS) antes y después del tratamiento de la incontinencia urinaria (IU). El objetivo fue evaluar la respuesta sexual y la calidad de vida de las pacientes con incontinencia urinaria antes y después del tratamiento. La muestra final contó con 27 pacientes. La media del score de ICIQ-SF pasó de 17 a 7 después del tratamiento $(\mathrm{p}<0,001)$ y la del score ponderado de FSFI no tuvo alteración en ningún dominio. Las pacientes con vejiga hiperactiva (VHA) presentaron puntajes ponderados menores de RS en todos los dominios. Las pacientes sometidas a cirugía presentaron mejoría de los puntajes ponderados en los dominios de: deseo $(\mathrm{p}=0,02)$, satisfacción $(\mathrm{p}=0,05)$ y el puntaje total $(\mathrm{p}=0,02)$. Trece pacientes con puntaje de 0 en el ICIQ-SF tuvieron un aumento en la media de los puntajes ponderados del FSFI en los dominios de: deseo $(p>0,01)$, satisfacción $(p=0,05)$ y puntaje total $(p=0,01)$. Se recomienda la evaluación de la respuesta sexual en pacientes con incontinencia urinaria, porque esta condición causa un impacto negativo en la calidad de vida (QoL).
\end{abstract}

Palabras claves: Incontinencia urinaria. Calidad de vida. Sexualidad.

\section{ABSTRACT}

FEMALE SEXUAL FUNCTION BEFORE AND AFTER TREATMENT OF URINARY INCONTINENCE

The prevalence of urinary incontinence is around $20 \%$ of healthy middle-aged women. Incontinence causes a negative impact on the quality of life and sexuality. From August 2002 to January 2004, 30 patients (mean age 43 years) with stress urinary incontinence (59\%), overactive bladder (15\%) and mixed incontinence (26\%) answered the ICIQ-SF(International Consultation on Incontinence Questionnarie-Short Form) and FSFI (Female Sexual Function Index) questionnaires before and after treatment for urinary incontinence. The follow - up ranged from 12 to 53 months. Mean ICIQ score was 17 and 7 before and after treatment respectivelly (p<0,001). Overactive bladder showed the worst scores in all domains. The patients who underwent surgery increased their scores of desire $(p=0,02)$, satisfaction $(p=0,05)$ and total score $(p=0,02)$. Thirteen patients which ICIQ was zero increased: desire $(p<0,01)$, satisfaction $(p=0,05)$ and total score $(p=0,01)$. Urinary incontinence significantly affects the quality of life. Therefore sexual evaluation in incontinence patients is recommended.

Keywords: Urinary incontinence. Quality of life. Sexuality. 
La incontinencia urinaria (IU) se define de acuerdo a la Sociedad Internacional de Incontinencia (ICS Internacional Continente Society) como "toda queja de pérdida urinaria ${ }^{1}$.

Es un problema de salud pública común en todo el mundo y su evolución tiene consecuencias psico-sociales que afectan cerca de 200 millones de personas ${ }^{2}$.

En los Estados Unidos (EUA) la prevalencia de la IU varía de 15 a $30 \%$ en la población general ${ }^{3}$.

Las mujeres son más afectadas por la IU que los hombres. De manera general, la prevalencia de incontinencia urinaria entre los hombres varía de 3 a $11 \%$ siendo de 10 a 58\% entre las mujeres $^{4}$. Con perjuicio significativo en los aspectos: doméstico y social de la vida de $20 \%$ de las mujeres con incontinencia urinaria ${ }^{5}$.

Los sintomas urinarios son perturbadores y causan un impacto negativo en la calidad de vida (GoL). Comprometen la vida diaria en los aspectos: domésticos, ocupacionales y físicos. Y las consecuencias de esta condición aumentan la incidencia de depresiones, neurosis y disfunciones sexuales ${ }^{6}$.

Actualmente, se recomienda el uso de instrumentos específicos que permiten evaluar el impacto de la incontinencia urinaria en diferentes poblaciones tanto desde el punto de vista global como específico, como es el caso de la investigación de la sexualidad ${ }^{7}$.

$\mathrm{El}$ presente estudio tiene por objetivo evaluar la calidad de vida y la respuesta sexual de pacientes con incontinencia urinaria antes y después del tratamiento por cirugía con cinchas antólogas, medicamentos anticolinérgicos y fisioterapia de entrenamiento de la musculatura del suelo pélvico.

\section{MATERIALES Y METODOS}

Se realizó un estudio prospectivo, que involucró a 30 pacientes portadoras de incontinencia urinaria (IU). La investigación se realizó en dos períodos: agosto 2002 a enero 2003 (fase de pretratamiento) y agosto 2003 a enero 2004 (fase post-tratamiento). El proyecto fue aprobado por el comité de ética en investigación.

La edad media fue de 43 años (31 a 51 años), con tiempo de inicio de los síntomas urinarios que varió de 12 a 53 meses. Se excluyeron las pacientes en período postmenopáusico, con cirugias previas para incontinencia urinaria, sin pareja fija, con alteraciones psiquiátricas o de la función cognitiva que incapacitase responder los cuestionarios.

Después de la evaluación clínica y urodinámica, las pacientes con IU fueron conducidas a una entrevista psicológica y las que cumplieron los criterios se incluyeron en el grupo de estudio. Todas las pacientes dieron su consentimiento para el estudio.

Participaron pacientes con: incontinencia urinaria de esfuerzo (59\%), vejiga hiperactiva (15\%) y con incontinencia urinaria mixta (26\%).

Las entrevistas fueron realizadas por el mismo encuestador y éste no participó de ninguno de los tipos de tratamiento y las pacientes respondieron a dos cuestionarios: ICIQ-SF y FSFI.

ICIQ-SF: International Consultation on Incontinence Questionnarie-Short Form que evalúa la calidad de vida en pacientes con incontinencia urinaria $^{8}$. Este instrumento posee 6 preguntas y los valores de tres de ellas sumados dan un puntaje (mínimo 3 máximo 21). Los puntajes finales fueron comparados antes y después del tratamiento.

FSFI: Female Sexual Function Index es un instrumento breve y multidimensional que evalúa la función sexual femenina9. Está compuesto por 19 preguntas que evalúan 5 dominios de la respuesta sexual (RS): deseo y estímulo subjetivo, lubricación, orgasmo, satisfacción y dolor o discomfort. Las puntuaciones individuales se obtienen por la suma de los ítems que comprenden cada dominio (puntajes simples), que son multiplicadas por el factor de ese dominio y dan el puntaje ponderado. La puntuación final (puntaje total: mínimo 2 y máximo 36 ) se obtiene por la suma de los puntajes ponderados de cada dominio. Se evaluó el puntaje de cada dominio y el puntaje total de la respuesta sexual (RS) de todas las pacientes. En seguida se analizaron los puntajes de la RS relacionada con el tipo de tratamiento: cirugía, farmacológico y fisioterapia. Las pacientes con puntaje final de ICIQ-SF igual a cero se analizaron por separado en cuanto a la RS. 
Para la evaluación estadística se realizó el análisis explorador inicial y en los casos en los que fue posible establecer comparaciones se utilizó el test de Wilcoxon para datos pareados con software Statistica ${ }^{\circledR}$. Los valores de $\mathrm{p}$ iguales o menores de 0,05 indicaron diferencia significativa en la RS antes y después del tratamiento de la IU.

\section{RESULTADOS}

Todas las pacientes habían completado por lo menos un grado de instrucción y 20 (66\%) eran económicamente activas. Del total, 26 (87\%) eran casadas y tenían una pareja única.

Todas las pacientes respondieron a los cuestionarios en la primera fase de evaluación. En la segunda fase, tres pacientes fueron excluidas del estudio porque no respondieron a los cuestionarios. La muestra final estuvo conformada por 27 pacientes.

La media del puntaje de ICIQ-SF general antes del tratamiento fue de $17( \pm 4,4)$ y después del tratamiento fue de $7( \pm 8,14)$. En esta evaluación, la diferencia antes y después del tratamiento fue estadísticamente significativa $(\mathrm{p}<0,01)$.

No hubo diferencias significativas en los puntajes ponderados de los dominios del FSFI evaluados antes y después del tratamiento de la IU, cuando se consideró como grupo único.

El mismo instrumento (FSFI) se utilizó para analizar por separado cada pregunta, clasificando a las pacientes en tres grupos distintos: empeoraron, se mantuvieron iguales o mejoraron después del tratamiento. Se observó que en la pregunta 13 (una de las que evalúa el orgasmo) y en las preguntas 14, 15 y 16 (que evalúan la satisfacción sexual) hubo menor índice de casos que empeoraron después del tratamiento (menos del 20\%).

La pregunta 6 (una de las que evalúa excitación subjetiva), la pregunta 8 (que evalúa la lubricación) y la pregunta 18 (que evalúa el dolor) tuvieron mayor número de respuestas con empeoramiento después del tratamiento.

\section{FSFI por tipo de incontinencia}

Dieciséis pacientes (59\%) tenían incontinencia urinaria de esfuerzo (IUE), siete (26\%) incontinencia mixta (IM) y cuatro (15\%) con vejiga hiperactiva (VHA).

FSFI de las pacientes con IUE

El deseo y la lubricación presentaron las peores medias del puntaje ponderado después del tratamiento. Sin embargo, las diferencias no fueron estadísticamente significativas en ninguno de los dominios estudiados.

FSFI de las pacientes con IM

$\mathrm{El}$ orgasmo presentó la peor media del puntaje ponderado después del tratamiento. Sin embargo, tampoco las diferencias fueron estadísticamente significativas en ninguno de los dominios evaluados.

FSFI de las pacientes con VHA

La lubricación y el dolor presentaron los peores puntajes ponderados después del tratamiento. Sin embargo, las diferencias no fueron estadísticamente significativas en ninguno de los dominios estudiados.

Al comparar las pacientes con otros tipos de incontinencia, las pacientes con VHA tuvieron menores puntajes ponderados de RS en todos los dominios, lo que indica una mayor insatisfacción con la vida sexual en general.

\section{FSFI por tipo de tratamiento}

Trece pacientes fueron intervenidas quirúrgicamente con cincha antóloga (48\%), 9 (33\%) de ellas recibieron tratamiento médico con anticolinérgicos y $5(18 \%)$ realizaron fisioterapia de entrenamiento de la musculatura del piso pélvico.

\section{FSFI de las pacientes que realizaron cirugía}

Las pacientes sometidas a cirugía presentaron una mejoría estadísticamente significativa de los puntajes ponderados en los dominios: deseo, satisfacción y puntaje total (Fig. 1).

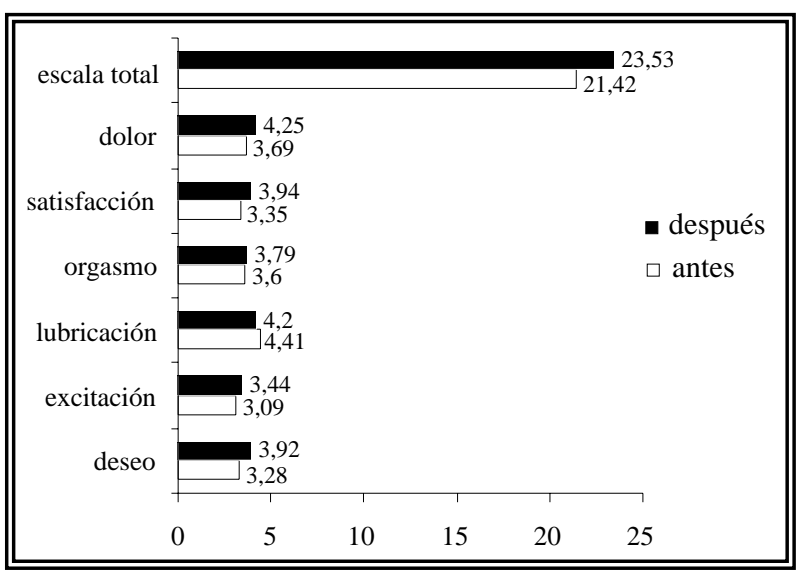

FIGURA 1: Puntajes ponderados de FSFI de las pacientes con se sometieron a cirugia. 


\section{FSFI antes y después del tratamiento farmaco-} lógico

El dominio de la satisfacción presentó un aumento de la media del puntaje ponderado después del tratamiento. Sin embargo, no hubo diferencias estadísticamente significativas en ninguno de los dominios estudiados.

\section{FSFI antes y después de la fisioterapia}

El dominio excitación presentó disminución de la media de puntaje ponderado después del tratamiento. Sin embargo, las diferencias no fueron estadísticamente significativas en ninguno de los dominios estudiados.

\section{ICIQ-SF combinado con FSFI}

Trece pacientes (48\%) presentaron resultados de ICIQ-SF con puntajes de cero, es decir, sin queja de incontinencia después del tratamiento (9 sometidas a cirugía (69\%), 3 tratadas con medicamentos (23\%), y 1 que se sometió a fisioterapia (7\%)). Los puntajes de estas pacientes tuvieron diferencias estadísticamente significativas en la media ponderada de los dominios deseo, satisfacción y en el puntaje total (Fig. 2).

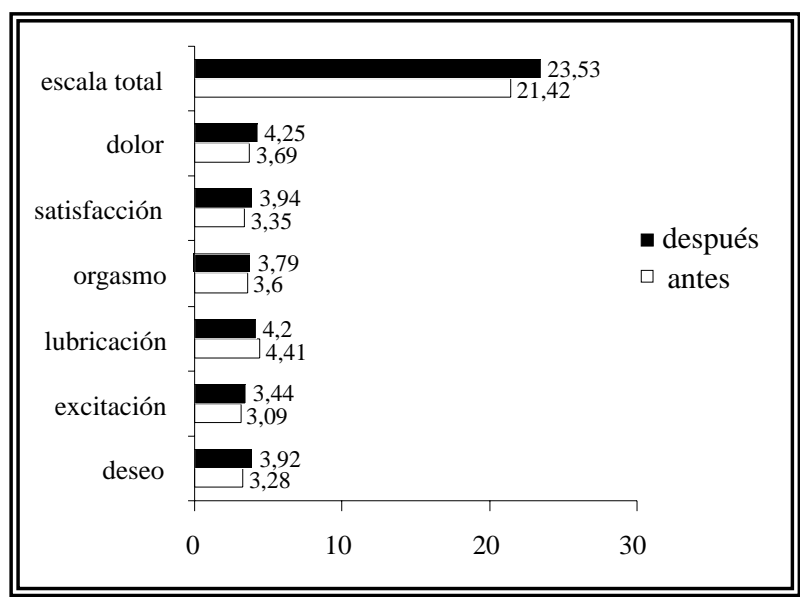

FIGURA 2: Puntajes ponderados de ICIQ-SF combinado con FSFI de las pacientes que mejoraron su RS.

\section{DISCUSIÓN}

La incontinencia urinaria es un problema común de las mujeres, actualmente, foco de una demanda significativa de programas sociales y discusión frecuente entre los sistemas de salud sobre la mejor prevención y terapéutica ${ }^{10}$.
Recientemente, los estudios también han abordado el problema de la GoL de las pacientes portadoras de $\mathrm{IU}^{11}$. Numerosos cuestionarios auto-aplicables, que proponen medidas objetivas del compromiso emocional de estas mujeres, pueden encontrarse en la literatura, aunque con resultados divergentes ${ }^{12}$.

El impacto de la incontinencia urinaria en la vida de las pacientes no está directamente relacionado al grado de pérdida de orina ${ }^{13}$, aunque algunos estudios han demostrado que mujeres con pérdida de orina menor tienen mejor tolerancia a su condición ${ }^{14}$.

La calidad de vida se define como la preocupación del individuo por su posición en un contexto cultural según sus valores, objetivos, expectativas y esto incluye las funciones físicas, psicológicas y sociales, y es recomendada en pacientes con incontinencia urinaria ${ }^{15}$.

Los problemas de salud se acompañan de sentimientos negativos de inferioridad, miedo, rabia, ansiedad y depresión. La amenaza de la salud y su significado para el paciente pueden afectar la calidad de sus relaciones interpersonales con familiares, cónyuges y amigos ${ }^{16}$.

Las enfermedades crónicas con sufrimiento prolongado afectan el humor y la autoestima y pueden alterar el ciclo de la respuesta sexual ${ }^{17}$.

En el pasado se pensaba que la incontinencia urinaria producía alteraciones emocionales que justificaban la presencia de disfunciones sexuales $^{18}$.

Hace dos décadas atrás, las evaluaciones de mujeres con pérdida urinaria ya referían los efectos adversos de esta condición en la sexualidad ${ }^{19}$. Aunque la incontinencia tiene un efecto negativo sobre la RS, pocas pacientes reciben información y tratamiento especializado ${ }^{20}$.

Como es un indicativo importante en la calidad de vida, se hizo necesaria su investigación.

$\mathrm{El}$ análisis de ICIQ-SF demostró una disminución significativa de la media del puntaje de 17 a 7 , que corresponde a la mejora en la calidad de vida (GoL) después del tratamiento para IU. Sin embargo, la evaluación de la RS obtenida por el FSFI no tuvo alteración significativa en ninguno de los dominios investigados. No hubo relación directa entre la mejoría de los síntomas urinarios y la RS de las pacientes en el grupo total. 
Para Bodden-Heirich (1999) los problemas uroginecológicos no son responsables de las quejas sexuales, Bassin un estado depresivo y ni con problemas uroginecológicos ${ }^{21}$. Otros autores concluyen que la satisfacción sexual no depende del diagnóstico ni del tratamiento de la incontinencia urinaria $^{22}$.

En cuanto a la evaluación de la RS relacionada a cada tipo de incontinencia, se observó que la IUE fue el tipo más frecuente con 17 casos, seguido de la IM y la $\mathrm{VHA}^{23}$.

En esta muestra hubo una mayor prevalencia de IUE en relación con las demás, así como está descrito en la literatura. Sin embargo, no se puede afirmar que las mujeres con esta queja tengan mayores alteraciones en la RS.

A pesar que las alteraciones de los puntajes ponderados no fueron significativas en ninguno de los dominios de FSFI, hubo disminución del deseo y de la lubricación en mujeres con IUE, disminución del orgasmo en aquellas con IM y disminución de la lubricación y del dolor en las que tenían VHA.

Se observaron puntajes ponderados más bajos en todos los dominios de la respuesta sexual de pacientes con VHA en comparación con otros tipos de incontinencia, lo que demuestra mayor insatisfacción con la vida sexual y en general.

Sin embargo, no existen datos en la literatura que justifiquen este hallazgo y deben realizarse más estudios.

No hubo alteración significativa en los puntajes ponderados de los dominios de la RS de las pacientes del grupo que recibió medicación y de aquellas con fisioterapia. Los estudios con mayor número de pacientes podrían mostrar resultados diferentes.

Las pacientes sometidas a cirugía (cincha), presentaron mejoría estadística significativa de los puntajes ponderados en los dominios: deseo, satisfacción, y puntaje total (Fig. 1).

En este grupo, es probable que la insatisfacción sexual estuviera relacionada con la IU e con la mejoría de los síntomas urinarios, mejoró la respuesta sexual. Además de esto, la resolución de la queja urinaria, sin la necesidad de tener que utilizar medicamentos o medidas de tratamiento continuas como la fisioterapia, puede devolver a las pacientes el estado de salud, mejo- rando su autoestima y contribuyendo a mejorar y retomar las relaciones sexuales satisfactorias.

En la literatura se encontraron datos divergentes. Un estudio con 55 mujeres sometidas a cincha pubo-vaginal con TVT (Tension free Vaginal Tape), encontró molestias sexuales en $20 \%$ de estas pacientes, de las cuales $15 \%$ fue dispareunia y $5 \%$ disminución de la libido ${ }^{24}$. Otro estudio con 67 mujeres sometidas a TVT encontró deterioro de la RS, con pérdida de la libido en $14 \%{ }^{25}$. Un estudio reciente realizado con mujeres incontinentes que fueron intervenidas quirúrgicamente, demostró mejoría subjetiva de la respuesta sexual, debido al aumento del deseo ${ }^{26}$.

Las pacientes que obtuvieron un puntaje de cero en el ICIQ, tuvieron una mejoría significativa en la RS, observada principalmente en los dominios de deseo, satisfacción y el puntaje total. Es decir, la cura de la IU resultó en mejoría de la RS.

\section{CONCLUSIONES}

Las pacientes sometidas a cirugía tuvieron mejoría en la respuesta sexual.

Se recomienda la evaluación de la respuesta sexual en mujeres con incontinencia urinaria porque esta condición, independientemente de su severidad, causa impacto negativo en la calidad de vida (GoL).

\section{REFERENCIAS}

1. Hunskaar S, Burgio K, Diokono A, Herzog AR, Hjalmas K, Lapitan MC. Epidemiology and Natural History of Urinary Incontinence in Women. Urology 2003;62(Suppl 4A):16-23.

2. Cayan S, Acar A, Bozlu M, Doruk E, Akhay E. Is Stress Urinary Incontinence a Risk Factor for Female Sexual Function? Eur Urology Supp 2003;2(1):194.

3. Parsons M, Cardoso L. The Classification of Urinary Incontinence. Rev Gynaecol Prac 2003;3(2):57-64.

4. Hunskaar S, Arnold EP, Burgio K, Herzog AR, Mallett VT. Epidemiology and natural history of urinary incontinence. In: Incontinence. Edited by Abrams, P; Khoury, S; Wein, A. London: Health Publicatioins 1999;199-226.

5. Burgio KL, Matthews KA, Engel BT. Prevalence, incidence and correlates of urinary incontinence in healthy middleaged women. J Urol 1991;146:1255-1259.

6. Parsons M, Cardoso L. The Classification of Urinary Incontinence. Rev Gynaecol Prac 2003;3(2):57-64.

7. Bradway C. Urinary incontinence among older women. Measurement of the effect on health-related quality of life. $\mathrm{J}$ Gerontol Nurs 2003;29(7):13-19.

8. Tamanini JTN, Dambros M, D’Ancona CAL, Palma PCR, Netto Jr NR. Validação para o português do International Consultation on Incontinence Questionnaire-Short Form (ICIQ-SF). Rev Saúde Pub 2004;38(3):438-444. 
9. Rosen R, Brown C, Heiman J, Leiblum S, Meston C, Shabsigh R, et al. The Female Sexual Function Index (FSFI): A Multidimensional Self-Report Instrument for the Assessment of Female Sexual Function. J Sex Marl Ther 2000;26(2):191-208.

10. Nihira MA, Henderson N. Epidemiology of urinary incontinence in women. Curr Womens Health Rep 2003;3(4):340347.

11. Hunskaar S, Burgio K, Diokno A, Herzog AR, Hjalmas K, Lapitan MC. Epidemiology and Natural History of Urinary Incontinence in Women. Urology 2003;62(Suppl 4A):16-23.

12. Symonds T. A Review of Condition-Specific Instruments to Assess the impact of Urinary Incontinence on Health-related Quality of Life. Euro Urol 2003;43(3):219-225.

13. Ozedogan N, Bejib NK, Yalcin O. Urinary Incontinence: Its prevalence, risk, factors and effects on the quality of life of women living in a region of Turkey. Gynaecol Obst Inv 2004;58(3): 145-150.

14. Giman JF, Harkins SW, Choi SC, Taylor JR, Fantl JA. Psychosocial impact of urinary incontinence in women. Obstet Gynecol 1987;70(3):378-381.

15. Kelleher CJ, Cardoso LD, Thozs-Hobson, PM. Quality of Life and Urinary Incontinence. Curr Opin Obst Gynaecol 1995; 7:404-408

16. Liberman JN, Hunt TL, Stewart WF, Wein A, Zhou Z, Herzog AR, et al. Health-related quality of life among adults with symptoms of overactive bladder: results from a U.S. community-based survey. Urology 2001;57(6):10441050.

17. Kaplan HS. A Nova Terapia do Sexo: tratamento dinâmico das disfunções sexuais. Tradução: Silva, OB. Rio de Janeiro. Nova Fronteira. 1977.

18. Stach-Lempinen B, Hakala AL, Laippala P, Lehtinen K, Metsanoja R, Kujansuu E. Severe depression determines quality of life in urinary incontinent women. Neurourol Urodyn 2003;22:563-568.
19. Hilton P. Urinary Incontinence During Sexual Intercourse: a common, but rarely volunteered, symptom. Brit J Obst Gynaecol 1988;95:377-381.

20. Thomas TM, Plymat KR, Blannin J Meade TW. Prevalence of urinary incontinence. Brith Med J 1980;281:1243-1245.

21. Bodden-Heidrich R, Beckmann MW, Libera B, Rechenberger IHG. Psychossomatic Aspects of Urinary Incontinence. Arch Gynecol Obstet 1999;262(3):151-158.

22. Barber MD, Visco AG, Giman JF, Fantl A, Bump RC. Sexual Function in Women with Urinary Incontinence and Pelvic Organ Prolapso. Obst Gynecol 2002;99(2):281-289.

23. Landers SJ. Incontinence treatable, if reported while millions have the condition, patients are often hesitant to talk about it. Health \& Science AM News. June 28, 2004.

24. Mazouni C, Karsenty G, Bretelle F, Bladou F, Gamerre M, Serment G. Urinary Complications and Sexual Function After the Tension-free Vaginal Tape Procedure. Acta Obst Gynecol Scan 2003;83(10):955-961.

25. Maaita M, Bhaumik J, Davies AE. Sexual function after using tension-free vaginal tape for the surgical treatment of genuine stress incontinence. BJU Int 2002;90(6):540543.

26. Pinto AC. O Impacto da Correção Cirúrgica da Incontinência Urinária aos Esforços pela Técnica de Suporte Suburetral na Vida Sexual de Mulheres Submetidas a esse Tratamento. São Paulo, 2003. (Tese - Doutorado - Universidade de São Paulo / USP).

Dr. P. Palma

E-mail: ppalma@uol.com.br

(Trabajo recibido el 1 de diciembre 2005) 